\title{
Il bilancio del fosforo e il danno d'organo
}

\author{
Antonio Bellasi \\ U.O.C. di Nefrologia e Dialisi, Azienda Ospedaliera Sant'Anna, Como \\ Dipartimento di scienze della salute, Università di Milano, Milano
}

\begin{abstract}
Phosphate balance and organ damage
Abstract. Numerous in vitro and clinical studies support the notion that phosphate and mineral bone metabolism abnormalities (known as chronic kidney disease-mineral and bone disorder, CKD-MBD) are closely associated with cardiovascular as well as bone diseases in CKD. Although the natural history and pathophysiology of mineral metabolism derangements are yet to be elucidated, it is widely accepted that the link between CKD-MBD and vascular calcification may explain the astonishing risk of unfavorable outcome connected to CKD-MBD. In this brief review article, we summarize the large existing body of evidence supporting the association between phosphate metabolism and organ damage as well as survival in CKD.
\end{abstract}

Key words: Phosphorus, Balance, Organ damage, Vascular calcifications, Mortality

Conflict of interest: AB has served on the Advisory Board and/or has been a speaker at symposia sponsored by AmgenDompé, Genzyme and/or Sanofi Sanifit

Ricevuto: 30 Luglio 2013; Accettato: 27 Agosto 2013

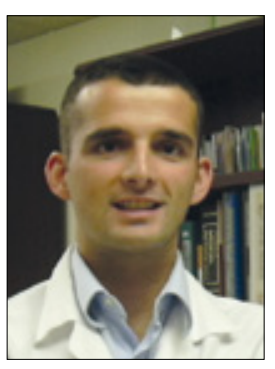

Antonio Bellasi

\section{Introduzione}

L'insufficienza renale cronica è considerata una vera e propria epidemia (1-3). Dati epidemiologici dimostrano come quasi un soggetto su dieci presenti alterazioni o della funzione renale o del sedimento urinario (1-3). Questa prevalenza, superiore a quella del diabete, sembra in espansione a causa sia dell'aumento dell'età media della popolazione generale che della diffusione di malattie cardiovascolari quali l'ipertensione arteriosa e il diabete. Numerosi lavori scientifici dimostrano che l'insufficienza renale cronica e le sue alterazioni metaboliche si associano a un significativo incremento del rischio di morte e di ospedalizzazione $(1,4)$. In considerazione del fatto che circa il $50 \%$ di questi eventi è di natura cardiovascolare, diversi esperti hanno proposto di considerare l'insufficienza renale come un equivalente della malattia coronarica. Tuttavia, nel recente passato, diversi studi dimostrano come, accanto ai fattori di rischio tradizionali, nell'insufficienza renale cronica esistano fattori di rischio specifici legati alla riduzione del filtrato glomerulare (chiamato anche GFR, che rappresenta una misura della funzione renale) (4). Tra questi ultimi, le alterazioni del metabolismo minerale sono state ampiamente associate alla malattia cardiovascolare e al rischio di prognosi infausta nell'insufficienza renale cronica (5). Obiettivo di questo articolo è di riassumere le evidenze scientifiche che suggeriscono un legame tra metabolismo del fosforo, malattia cardiovascolare (calcificazioni vascolari) e rischio di morte nei vari stadi dell'insufficienza renale cronica.

\section{Bilancio del fosforo e iperfosforemia}

Il bilancio del fosforo risulta sostanzialmente dall'equilibrio tra quantità di fosforo ingerita con la dieta, dalla quota scambiata dall'osso (il magazzino del fosforo corporeo) e dalla quantità escreta con le urine e le feci nelle 24 ore (6). Si ritiene che il contenuto di fosforo di una dieta standard in un paese occidentale contenga all'incirca 1-1.5 g/die (6). Analogamente, nel corso di una giornata, circa 150-200 mg/die sono scambiati (rimossi e depostati) nel tessuto osseo, circa 400$600 \mathrm{mg} /$ die vengono eliminati con le feci e la restante parte in eccedenza viene eliminata con le urine (circa 700-800 mg/die) (6). Esistono diversi meccanismi preposti alla fine regolazione dell' escrezione urinaria dei fosfati in eccesso (PTH, Fibrobast factor 23, altre fosfatonine) che ancora oggi conosciamo solo in parte e di cui, nel prossimo futuro, dovranno essere chiariti numerosi aspetti del metabolismo (6).

Con la riduzione della funzione renale, avviene una progressiva riduzione delle unità funzionali del rene (nefroni) e, conseguentemente, della capacità di modulare l'eliminazione della quota in eccesso di fosfati proveniente dalla dieta. Diversi studi epidemiologici che hanno studiato il timing della comparsa delle diverse complicanze metaboliche che caratterizzano il 
corso dell'insufficienza renale cronica hanno, tuttavia, evidenziato come l'iperfosfatemia sia una complicanza abbastanza tardiva (7-9). Si ritiene, infatti, che l'iperfosforemia sia una condizione prevalente solo tra i soggetti con un filtrato glomerulare (GFR) pari o inferiore a $30 \mathrm{~mL} / \mathrm{min} / 1.73 \mathrm{~m}^{2}$ (IRC stadio 3b-4, secondo la classificazione della National Kidney Foundation, NKF-KDOQI) (7-9).

Se, tuttavia, invece di considerare il siero, valutiamo l'escrezione urinaria di fosforo (9), si nota come la fosfaturia sia significativamente incrementata quando ancora i valori di fosforemia sono contenuti all'interno del range di normalità e per valori di GFR molto superiori a $30 \mathrm{~mL} / \mathrm{min} / 1.73 \mathrm{~m}^{2}$ (9). Sia che si consideri la fosfaturia delle urine delle 24 ore sia che si consideri la frazione di escrezione del fosforo, un lavoro di Craver et al. (9) dimostra che l'escrezione di fosforo è significativamente incrementata già nelle prime fasi dell'insufficienza renale cronica (CKD stadio 2) (9). Diversi fattori umorali sono responsabili della fine regolazione dell'escrezione urinaria di fosforo. Tra questi oggi riconosciamo il paratormone (PTH) e il fibroblast growth factor 23 (FGF23), che si trovano in elevate concentrazioni nel siero dei pazienti esposti a un bilancio fosforico positivo e che, anche se non sono stati chiariti nel dettaglio i meccanismi che ne regolano la secrezione, sono in grado di indurre un incremento dell'eliminazione urinaria di fosforo $(8,9)$.

In sintesi, il bilancio del fosforo (piuttosto che la fosforemia) è alterato fin dalle prime fasi dell'insufficienza renale. Per mantenere la fosforemia all'interno del range di normalità esistono diversi meccanismi, operanti anche per modeste riduzioni del filtrato glomerulare, responsabili di un precoce incremento dell'escrezione dei fosfati. Come vedremo in seguito, tuttavia, questi meccanismi adattativi sono legati al danno d'organo e, verosimilmente, contribuiscono al danno d'organo associato a un bilancio positivo del fosforo.

\section{Quali sono i meccanismi che legano il metabolismo del fosforo al danno d'organo}

Diversi lavori documentano una stretta e forte associazione tra il metabolismo del fosforo, il sistema renina angiotensina $(10,11)$ e diversi parametri di danno o malattia cardiovascolare, quali le calcificazioni vascolari (12), l'aterosclerosi (13), l'ipertrofia ventricolare sinistra $(14,15)$ e la disfunzione endoteliale (16). Anche se questi dati sono derivati da studi osservazionali e non randomizzati, queste evidenze sono oggi ritenute le possibili spiegazioni dell'incremento del rischio di morte associato alle alterazioni del metabolismo del fosforo.

In una recente analisi post hoc dello studio Rein (17), è stato descritto un forte legame tra i livelli di fosforemia e il rischio di progressione dell'insufficienza renale cronica (definito come raddoppio della creatinina o come necessità di iniziare il trattamento dialitico) tra i pazienti con insufficienza renale cronica e proteinuria superiore al grammo/die. Se questo dato non è nuovo in quanto documentato da diversi studi clinici (17), la novità di questo lavoro consiste nell'osservazione che i soggetti con iperfosforemia (valori superiori a $4.6 \mathrm{mg} / \mathrm{dL}$ ) all'ingresso nello studio non sembrano protetti

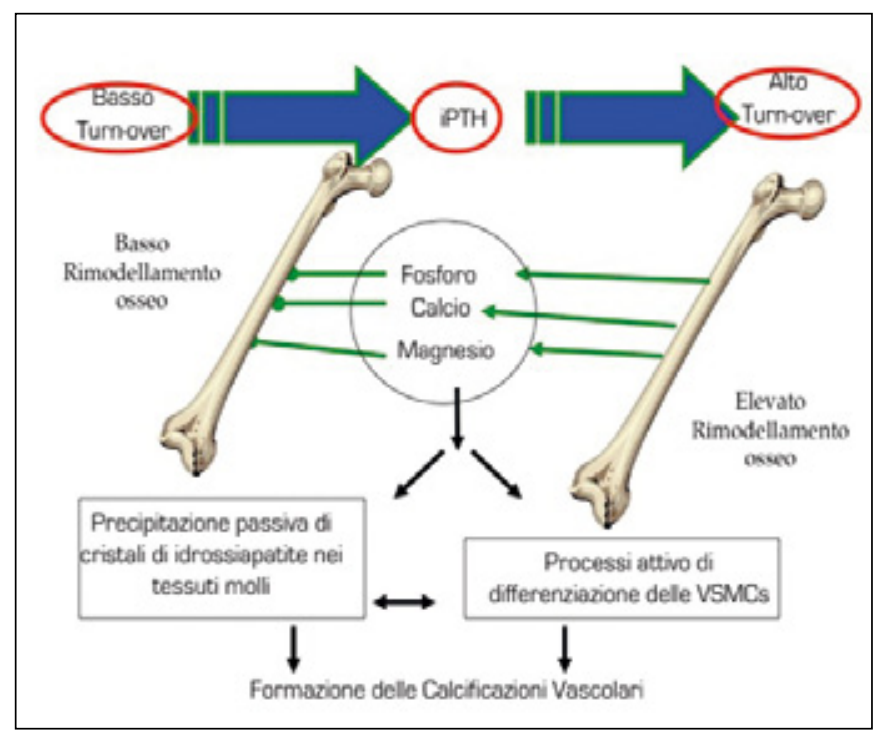

Fig. 1 - Relazione tra osso e vasi. Sia i quadri di osteodistrofia a basso turn-over che quelli ad alto turn-over sono caratterizzati dall'accumulo in circolo di metaboliti in grado di attivare il procedimento di formazione delle calcificazioni vascolari mediato sia da meccanismi attivi che da meccanismi passivi (VSMC: cellule della muscolatura liscia della parete arteriosa).

dalla terapia con ACE inibitori (ACE-i), come invece documentato per i pazienti con valori di fosforo all'interno del range di normalità (17). Il legame tra fosforo e proteinuria è emerso anche in uno studio successivo nel quale Di Iorio et al. (18) hanno dimostrato che l'effetto antiproteinurico della dieta ipoproteica si riduceva significativamente per valori di fosforemia o fosfaturia progressivamente più elevati (test di interazione altamente significativo) in una coorte di 99 pazienti con $\mathrm{CKD}$ stadio $3 \mathrm{~B}$ e proteinuria non controllata dalla terapia farmacologica standard (ACE-i o antagonisti del recettore dell'angiotensina) (18). Se questi dati non permettono di evidenziare quali siano i meccanismi che legano il pool del fosforo corporeo all'azione antiproteinurica di questi presidi terapeutici, abbiamo numerose segnalazioni di studi in vitro e su modelli animali che suggeriscono uno stretto legame tra sistema renina-angiotensina e metabolismo del fosforo (10).

Sebbene esistano diversi tipi di calcificazioni vascolari (12), oggi abbiamo una ragionevole sicurezza in merito al fatto che le alterazioni del metabolismo minerale siano legate alle calcificazioni che si formano a livello della tonaca muscolare della parete arteriosa. Anche in questo caso i meccanismi sono stati chiariti solo parzialmente. Tuttavia, studi condotti su cellule della muscolatura liscia della parete arteriosa (VSMC) dimostrano come queste cellule vadano incontro a un processo di differenziamento cellulare quando sono coltivate con concentrazioni crescenti di fosforo e calcio (1921). Infatti, le VSMC perdono le caratteristiche fenotipiche delle cellule muscolari e acquisiscono le caratteristiche di cellule simili agli osteoblasti (19-21). Acquisiscono, quindi, la capacità di secernere matrice cellulare tipica del tessuto 
osseo innescando i meccanismi che portano alla formazione delle calcificazioni vascolari (19-21). Numerose evidenze epidemiologiche legano, inoltre, il sistema cardiovascolare al tessuto osseo (12). Nell'insufficienza renale cronica, sia gli stati a elevato che quelli a basso turn-over osseo potrebbero essere associati a un accumulo in circolo di fosfati, calcio, magnesio e altri minerali, che porterebbe alla formazione delle calcificazioni vascolari sia per deposizione passiva di cristalli di idrossiapatite che per induzione di processi di sdifferenziazione delle cellule della tonaca muscolare delle arterie (Fig. 1) (12).

Anche se non è ancora stato chiarito se la calcificazione vascolare è un marker o un effettore di danno cardiovascolare, una vasta letteratura medica associa la presenza e l'estensione delle calcificazioni vascolari al rischio di morte o di eventi cardiovascolari sia nella popolazione generale (22) che tra i soggetti con insufficienza renale $(23,24)$. In particolare, tra i soggetti sottoposti a un trattamento sostitutivo cronico, l'assenza di calcificazione corrisponde a una probabilità di sopravvivenza del $90 \%$ a 3 anni (25).

Dati dello studio Aric suggeriscono che il metabolismo del fosforo sia legato anche all'estensione della Placca aterosclerotica (13), misurata mediante la valutazione dello spessore intima-media delle carotidi. Da notare che questa relazione è statisticamente significativa anche tra soggetti con funzione renale normale e per valori di fosforemia all'interno del cosiddetto range di normalità, suggerendo, forse, che la fosforemia non sia un valido marker del bilancio o del pool corporeo del fosforo (13).

Il fibroblast growth factor 23 (FGF23), fattore fosfaturico e corresponsabile dell'incremento della fosfaturia fin dalle prime fasi dell'insufficienza renale (8), oltre a regolare il bilancio del fosforo, potrebbe mediare il danno d'organo. In un recente lavoro, Faul et al. (15) dimostrano che, all'aumentare dei livelli circolanti di FGF23, aumenta la probabilità di sviluppare ipertrofia ventricolare sinistra (15). Da notare che, in questo lavoro, i meccanismi responsabili dell'incremento della massa cardiaca sono indipendenti dalla pressione arteriosa, dai livelli sierici del fosforo e dalla presenza di klotho (il corecettore di FGF23) (15). Analogamente anche il carico di fosforo derivante dalla dieta è stato associato a un incremento della massa cardiaca, indipendentemente dall'aggiustamento per diversi fattori associati al metabolismo del fosforo o alla massa cardiaca (14).

Infine, l'incremento del FGF23 sierico potrebbe essere legato alla disfunzione endoteliale, altro marker di danno d'organo e predittore di eventi cardiovascolari. In una coorte di 183 pazienti con insufficienza renale cronica allo stadio $3-4$, l'incremento dei livelli circolanti di FGF23 si associava a una minore risposta endoteliale allo stimolo ischemico (16). Sebbene tutti questi dati documentino uno stretto legame tra il sistema cardiovascolare e il metabolismo del fosforo, a oggi non esiste uno studio di intervento che dimostri che la riduzione dei livelli sierici di fosforo o del bilancio del fosforo migliori significativamente le alterazioni di questi marker di danno d'organo. Tuttavia, si ritiene che queste evidenze possano spiegare lo stretto legame tra fosforemia e rischio di progressione dell'insufficienza renale o di morte.

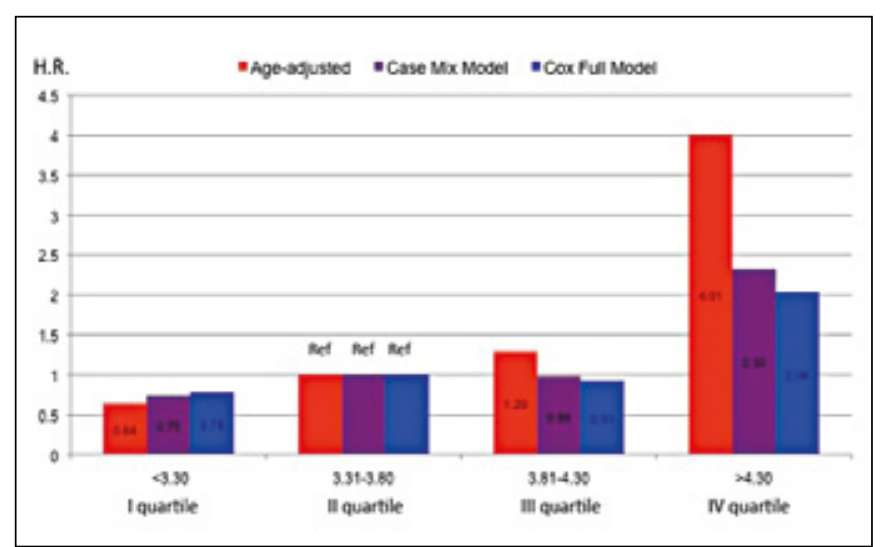

Fig. 2 - Relazione tra livelli ematici di fosforo (in ascissa sono rappresenatati i quartili della distribuzione del fosforo sierico) e rischio di sviluppare l'endpoint composito (In ascissa è rappresentato l'Hazard Ratio morte per il rischio di morte ogni causa o ingresso in dialisi). Il gruppo di riferimento è il quartile di fosforo con valori sierici compresi tra 3.3$3.8 \mathrm{mg} / \mathrm{dL}$. Analisi aggiustate per età (barre rosse), età e case mix (barre viola), età, case mix e emoglobina, calcio sierico, acido urico, uso di ACE inibitori, vitamina D e sali di calcio (barre blu).

\section{Bilancio del fosforo, manipolazione del fosforo e prognosi}

Nell'ultimo decennio, diversi lavori hanno analizzato il legame tra i livelli di fosforo e il rischio di morte per ogni causa o di progressione dell'insufficienza renale cronica verso gli stadi terminali della malattia renale (26). Sebbene non tutti abbiano confermato questa relazione (27), sembra che l'incremento dei valori di fosforo anche all'interno del range di normalità si accompagni a un significativo incremento del rischio di morte o di ingresso in dialisi (26). In uno studio italiano (5), abbiamo dimostrato che, in una coorte di 1716 pazienti con insufficienza renale cronica di grado moderato (filtrato glomerulare mediano $25 \mathrm{~mL} / \mathrm{min}$ ), valori di fosforo superiori a $4.3 \mathrm{mg} / \mathrm{dL}$ si associavano a un significativo rischio dell'evento composito morte per ogni causa o ingresso in dialisi (5). Questa associazione era indipendente da diversi fattori associati al metabolismo minerale o agli endpoint considerati (5). Anche se la relazione diventava significativa da un punto di vista statistico per valori di fosforo sierico superiori a $4.3 \mathrm{mg} / \mathrm{dL}$ (Fig. 2), esistono diversi altri lavori che suggeriscono che questa associazione può essere presente anche per valori di fosforemia inferiori e contenuti nel range di normalità (26). Tuttavia, la scarsa accuratezza della fosforemia come marker di bilancio del fosforo potrebbe giustificare questi risultati discrepanti. Un lavoro pubblicato a inizio anno da Sim et al. (28) ha descritto, in una vasta coorte di 94.989 soggetti seguita per 11 anni, un significativo incremento del rischio di morte renale associato a valori di fosforo sierico progressivamente crescenti. A ogni incremento di $0.5 \mathrm{mg} / \mathrm{dL}$ della fosforemia si associava un incremento del $40 \%$ di progressione dell'insufficienza renale fino alla dialisi (Hazard Ratio: 1.40; Intervallo di Confidenza al 95\%: 1.06-1.84) (28). 
Tutti questi dati suggeriscono che, manipolando il bilancio del fosforo, si può migliorare la prognosi dei soggetti affetti da insufficienza renale cronica. Sebbene, a oggi, manchi uno studio clinico randomizzato in grado di rispondere in modo definitivo a questo interrogativo, abbiamo oggi diverse segnalazioni circa la potenziale tossicità di alcuni trattamenti dell'iperfosforemia (29). Attualmente i principali presidi terapeutici dell'iperfosforemia sono rappresentati dalla dieta ipoproteica/ipocalorica e dall'uso di chelanti del fosforo (6). Questi ultimi legano il fosforo alimentare nel lume dell'intestino e ne impediscono l'assorbimento. Esistono diversi medicinali, anche se, attualmente, $i$ chelanti più usati sono i sali di calcio e il sevelamer (6). Il sevelamer è un polimero che non è assorbito a livello intestinale e che potrebbe avere degli effetti additivi come la riduzione dei lipidi sierici, dell'acido urico, di qualche tossina uremica (cresolo, indoxil solfato) e di marker di fase acuta (p. es., proteina $\mathrm{C}$ reattiva), oltre alla riduzione dell'assorbimento di fosforo (30). Tuttavia, la reale superiorità di questo farmaco è stata oggetto di lunghe discussioni nel recente passato (31). Di Iorio et al. (32) hanno testato l'impatto di sevelamer e calcio carbonato sull'endpoint composito morte per ogni causa o ingresso in dialisi in una coorte di 239 pazienti con iperfosforemia e insufficienza renale cronica di grado moderato ma non in trattamento dialitico cronico (creatinina clearance $32 \mathrm{~mL} / \mathrm{min}$ ). Al completamento dei 36 mesi di follow-up previsti, i soggetti trattati con sale di calcio presentavano un rischio significativo di sviluppare l'endpoint composito rispetto ai soggetti trattati con sevelamer (32). Analogamente, il rischio di morte per ogni causa, di ingresso in dialisi e di sviluppare calcificazioni vascolari sembrava ridotto tra i soggetti allocati al trattamento con sevelamer (32).

Sebbene non tutti gli studi clinici a oggi pubblicati abbiano fornito gli stessi risultati, una recentissima metanalisi conclude per una significativa riduzione del rischio di morte (rischio relativo: 0.78; Intervallo di Confidenza al 95\%: 0.61-0.98) e di progressione delle calcificazioni vascolari associato all'uso di chelanti del fosforo non a base di sali di calcio (29). Anche se, a oggi, non è possibile rispondere con certezza alla domanda se la riduzione del bilancio fosforico possa migliorare la prognosi, sembra che l'evidenza disponibile suggerisca di non usare chelanti a base di calcio.

\section{Conclusioni}

La descrizione della stretta associazione tra le alterazioni del metabolismo minerale e del fosforo, l'osteodistrofia renale e le calcificazioni vascolari tra $\mathrm{i}$ soggetti con insufficienza renale ha generato un grande interesse per la storia naturale e la patogenesi di queste alterazioni metaboliche. Oggi, si ritiene che tutte queste alterazioni siano aspetti della medesima sindrome, chiamata Chronic Kidney Disease Mineral Bone Disorder (CKD-MBD), e che contribuiscano a condizionare, insieme ad altri fattori di rischio, la prognosi dei pazienti affetti da insufficienza renale cronica (33). Un crescente numero di pubblicazioni lega, infatti, il metabolismo del fosforo e le calcificazioni vascolari al rischio di morte o di progressione dell'insufficienza renale cronica fino alla dialisi $(12,22,26)$. Tuttavia, sebbene esistano evidenze che la manipolazione del bilancio del fosforo possa avere delle ripercussioni sulla prognosi (29), a oggi manca lo studio clinico randomizzato in grado di rispondere in modo definitivo al quesito se la riduzione del bilancio del fosforo possa migliorare la prognosi dei soggetti affetti da insufficienza renale cronica. In considerazione dell'espansione della popolazione con alterazioni della funzione renale e dei costi connessi con la gestione delle complicanze metaboliche dell'insufficienza renale cronica, questo studio rappresenta una priorità per la comunità nefrologica.

\section{Riassunto}

Numerose evidenze cliniche e di laboratorio suggeriscono una relazione stretta tra le alterazioni del metabolismo minerale (oggi chiamate Chronic Kidney Disease Mineral Bone Disorders, CKD-MBD) e i marker di danno cardiovascolare e di malattia ossea nell'insufficienza renale cronica. Anche se i meccanismi non sono stati ancora completamente chiariti, si ritiene che l'associazione con la malattia cardiovascolare, in particolare con le calcificazioni vascolari, rappresenti una valida spiegazione dell'incremento del rischio di morte o di progressione verso l'insufficienza renale cronica terminale associata alle alterazioni del metabolismo minerale. In questo articolo si vogliono riassumere le evidenze scientifiche che suggeriscono il legame tra metabolismo del fosforo, danno d'organo e sopravvivenza nell'insufficienza renale cronica.

Parole chiave: Fosforo, Bilancio, Danno d'organo, Calcificazioni vascolari, Mortalità

Dichiarazione di conflitto di interesse: $\mathrm{AB}$ ha partecipato ad advisory Board e/o è stato relatore a simposi sponsorizzati da Amgen-Dompè, Genzyme, Sanofi Sanifit
Indirizzo degli Autori:

Dr. Antonio Bellasi

Via Ravona 20

22020 San Fermo della Battaglia (CO)

antonio.bellasi@hsacomo.org 


\section{Bibliografia}

1. Go AS, Chertow GM, Fan D, McCulloch CE, Hsu CY. Chronic kidney disease and the risks of death, cardiovascular events, and hospitalization. N Engl J Med 2004; 351: 1296-305.

2. Meguid El Nahas A, Bello AK. Chronic kidney disease: the global challenge. Lancet 2005; 365: 331-40.

3. Coresh J, Astor BC, Greene T, Eknoyan G, Levey AS. Prevalence of chronic kidney disease and decreased kidney function in the adult US population: Third National Health and Nutrition Examination Survey. Am J Kidney Dis 2003; 41: 1-12.

4. Foley RN, Murray AM, Li S, et al. Chronic kidney disease and the risk for cardiovascular disease, renal replacement, and death in the United States Medicare population, 1998 to 1999. J Am Soc Nephrol 2005; 16: 489-95.

5. Bellasi A, Mandreoli M, Baldrati L, et al. Chronic Kidney Disease Progression and Outcome According to Serum Phosphorus in Mild-to-Moderate Kidney Dysfunction. Clin J Am Soc Nephrol 2011; 6: 883-91.

6. Bellasi A, Galassi A, Cozzolino M, Di Iorio B. The evolving world of Chronic Kidney Disease Mineral Bone Disorder (CKD-MBD). EMJ Neph 2013; 1: 20-31.

7. Moranne O, Froissart M, Rossert J, et al. Timing of onset of CKD-related metabolic complications. J Am Soc Nephrol 2009; 20: 164-71.

8. Isakova T, Wahl P, Vargas GS, et al. Fibroblast growth factor 23 is elevated before parathyroid hormone and phosphate in chronic kidney disease. Kidney Int 2011; 79: 1370-8.

9. Craver L, Marco MP, Martinez I, et al. Mineral metabolism parameters throughout chronic kidney disease stages 1-5--achievement of K/DOQI target ranges. Nephrol Dial Transplant 2007; 22: 1171-6.

10. Cozzolino M, Gentile G, Mazzaferro S, Brancaccio D, Ruggenenti P, Remuzzi G. Blood Pressure, Proteinuria, and Phosphate as Risk Factors for Progressive Kidney Disease: A Hypothesis. Am J Kidney Dis. 2013 May 9. pii: S0272-6386(13)00678-1. doi: 10.1053/j.ajkd.2013.02.379. [Epub ahead of print]

11. de Borst MH, Vervloet MG, ter Wee PM, Navis G. Cross talk between the renin-angiotensin-aldosterone system and vitamin D-FGF-23-klotho in chronic kidney disease. J Am Soc Nephrol 2011; 22: 1603-9.

12. Raggi P, Giachelli C, Bellasi A. Interaction of vascular and bone disease in patients with normal renal function and patients undergoing dialysis. Nat Clin Pract Cardiovasc Med 2007; 4: 26-33.

13. Onufrak SJ, Bellasi A, Shaw LJ, et al. Phosphorus levels are associated with subclinical atherosclerosis in the general population. Atherosclerosis 2008; 199: 424-31.

14. Yamamoto KT, Robinson-Cohen C, de Oliveira MC, et al. Dietary phosphorus is associated with greater left ventricular mass. Kidney Int 2013; 83: 707-14.

15. Faul C, Amaral AP, Oskouei B, et al. FGF23 induces left ventricular hypertrophy. J Clin Invest 2011; 121: 4393-408.

16. Yilmaz MI, Sonmez A, Saglam M, et al. FGF-23 and vascular dysfunction in patients with stage 3 and 4 chronic kidney disease. Kidney Int 2010; 78: 679-85.
17. Zoccali C, Ruggenenti P, Perna A, et al. Phosphate may promote CKD progression and attenuate renoprotective effect of ACE inhibition. J Am Soc Nephrol 2011; 22: 1923-30.

18. Di Iorio BR, Bellizzi V, Bellasi A. Phosphate attenuates the anti-proteinuric effect of very low-protein diet in CKD patients. Nephrol Dial Transplant 2013; 28: 632-40.

19. Li X, Yang HY, Giachelli CM. Role of the Sodium-Dependent Phosphate Cotransporter, Pit-1, in Vascular Smooth Muscle Cell Calcification. Circ Res 2006; 98 (7): 905-12.

20. Jono S, McKee MD, Murry CE, et al. Phosphate regulation of vascular smooth muscle cell calcification. Circ Res 2000; 87: E10-7.

21. Reynolds JL, Joannides AJ, Skepper JN, et al. Human vascular smooth muscle cells undergo vesicle-mediated calcification in response to changes in extracellular calcium and phosphate concentrations: a potential mechanism for accelerated vascular calcification in ESRD. J Am Soc Nephrol 2004; 15: 2857-67.

22. Bellasi A, Lacey C, Taylor AJ, et al. Comparison of prognostic usefulness of coronary artery calcium in men versus women (results from a meta- and pooled analysis estimating all-cause mortality and coronary heart disease death or myocardial infarction). Am J Cardiol 2007; 100: 409-14.

23. Block GA, Raggi P, Bellasi A, Kooienga L, Spiegel DM. Mortality effect of coronary calcification and phosphate binder choice in incident hemodialysis patients. Kidney Int 2007; 71: 438-41.

24. Watanabe R, Lemos MM, Manfredi SR, Draibe SA, Canziani ME. Impact of cardiovascular calcification in nondialyzed patients after 24 months of follow-up. Clin J Am Soc Nephrol 2010; 5: 189-94.

25. Bellasi A, Raggi P. Vascular imaging in chronic kidney disease. Curr Opin Nephrol Hypertens 2012; 21: 382-8.

26. Bellasi A, Cozzolino M, Adragao T, Di Iorio B, Russo D. Phosphate binders in moderate chronic kidney disease: where do we stand? J Nephrol 2013: 0.

27. Mehrotra R, Peralta CA, Chen SC, et al. No independent association of serum phosphorus with risk for death or progression to end-stage renal disease in a large screen for chronic kidney disease. Kidney Int 2013.

28. Sim JJ, Bhandari SK, Smith N, et al. Phosphorus and risk of renal failure in subjects with normal renal function. Am J Med 2013; 126: 311-8.

29. Jamal SA, Vandermeer B, Raggi P, et al. Effect of calcium-based versus non-calcium-based phosphate binders on mortality in patients with chronic kidney disease: an updated systematic review and meta-analysis. Lancet 2013.

30. Evenepoel P. Control of hyperphosphatemia beyond phosphate. Kidney Int 2007; 71: 376-9.

31. Palmer SC, Craig JC, Strippoli GF. Sevelamer: a promising but unproven drug. Nephrol Dial Transplant 2007; 22: 2742-5.

32. Di Iorio B, Bellasi A, Russo D. Mortality in kidney disease patients treated with phosphate binders: a randomized study. Clin J Am Soc Nephrol 2012; 7: 487-93.

33. Moe S, Drueke T, Cunningham J, et al. Definition, evaluation, and classification of renal osteodystrophy: a position statement from Kidney Disease: Improving Global Outcomes (KDIGO). Kidney Int 2006; 69: 1945-53. 